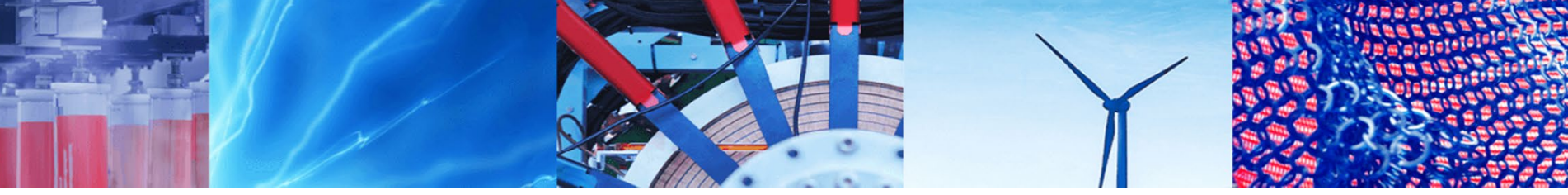

Research Article

\title{
Real-time 2D-3D door detection and state classification on a low-power device
}

\author{
João Gaspar Ramôa ${ }^{1,2}$ (V) Vasco Lopes ${ }^{1,2} \cdot$ Luís A. Alexandre $^{1,2} \cdot$ S. Mogo ${ }^{2}$
}

Received: 17 July 2020 / Accepted: 17 April 2021

Published online: 29 April 2021

(c) The Author(s) $2021 \quad$ OPEN

\begin{abstract}
In this paper, we propose three methods for door state classification with the goal to improve robot navigation in indoor spaces. These methods were also developed to be used in other areas and applications since they are not limited to door detection as other related works are. Our methods work offline, in low-powered computers as the Jetson Nano, in real-time with the ability to differentiate between open, closed and semi-open doors. We use the 3D object classification, PointNet, real-time semantic segmentation algorithms such as, FastFCN, FC-HarDNet, SegNet and BiSeNet, the object detection algorithm, DetectNet and 2D object classification networks, AlexNet and GoogleNet. We built a 3D and RGB door dataset with images from several indoor environments using a 3D Realsense camera D435. This dataset is freely available online. All methods are analysed taking into account their accuracy and the speed of the algorithm in a low powered computer. We conclude that it is possible to have a door classification algorithm running in real-time on a low-power device.
\end{abstract}

Keywords Door detection - Door state classification - Door segmentation · Jetson nano · 2D-3D Door dataset · RealTime

\section{Introduction}

New mobile robots with better components and software are built daily for several purposes, from smart vacuum cleaners, [1], delivery robots, [2], security robots, [3], nursing assistant systems, [4] to intelligent housekeepers, [5], that help people with difficulties in their daily tasks.

Door detection and its state classification (we consider three possible states: closed, open or semi-open) are crucial for this type of intelligent systems to safely navigate in indoor spaces. Usually, the task of these systems implies moving between rooms and dealing with doors. It is required to provide the robot with the necessary information about the door so it can safely navigate between rooms without any problem.
Door state classification is not restricted to mobile robots and robotics, it can be applied to other problems and areas like helping visually impaired people to safely move between rooms by providing information about the existing doors and their status.

In this paper, we propose three methods for door state classification, where each one uses different information: 1) only 3D information; 2) 3D and 2D (RGB) information; and 3) only $2 \mathrm{D}(\mathrm{RGB})$ information. We focus on an approach that works in low-power systems such as the single-board computers Nvidia Jetson Nano or the Raspberry Pi. Our methods work in real-time, despite running in low-power systems with weak GPU and are based in 2D and 3D object classification, 2D object detection and 2D semantic segmentation methods. We improved our

$\bowtie$ João Gaspar Ramôa, gaspar.gomes@ubi.pt; Vasco Lopes, vasco.lopes@ubi.pt; Luís A. Alexandre, luis.alexandre@ubi.pt; S. Mogo, sipmogo@gmail.com | ${ }^{1}$ NOVA LINCS, Costa da Caparica, Portugal. ${ }^{2}$ Universidade da Beira Interior, Rua Marquês d' Avila e Bolama, 6201-001 Covilhã, Portugal. 
previous work dataset [6], with 3D and RGB images with three different state classes: open doors, closed doors and semi-open doors. The images were captured using a 3D Realsense Camera. The developed methods were compared in terms of test accuracy and inference speed. We used a single board computer equipped with a 3D camera and powered by a power-bank. This mobile system was used for testing the speed of our methods.

The focus of this work was in the door detection and state classification algorithms, without concerning about the rest of the robot hardware. The majority of the methods, focus on door detection only, without having to classify its state, and in some cases door handle detection for robot grasping. We propose that if the door state is classified as closed, the robot must call a human for help. If it is open the robot can simply go through it and if it is semi-open, the robot can either get around it or try to open it simply by gently pushing it. The advantage of our approach is that allows easier integration in different robot structures with different dimensions by classifying the door state with different thresholds. Our method was also tested in our developed dataset which represents more real world scenarios with more difficult cases such as obscurations, blur images, varying light conditions and different door textures.

In short, the contributions of the paper are:

- We propose three different door state classification methods, where each one uses different types of information, and all are capable of working in real-time in low-power systems.

- A labelled dataset with RGB and depth images of closed, open and semi-open doors for 2D and 3D state door classification.

- A dataset for 2D door segmentation with annotated doors and door frames.

- A dataset for 2D door detection properly annotated.

The remainder of this paper is structured as follows: Section 2 does an overview of the state-of-the-art. Section 3 describes the door state classification and detection problem. Section 4 describes the proposed methods for door classification. Section 5 describes the dataset built. Section 6 describes the experiments and results of our methods. Section 7 presents the conclusions and future work.

\section{Related work}

There are already a vast number of studies that used door detection and classification for robot navigation tasks as moving between rooms, robotic handle grasping and others. Some have used sonar sensors with visual information,
[7, 8], others used only colour and shape information, [9], or just 3D shape information, [10], some have used simple feature extractors, $[11,12]$ and others have used more modern methods like CNN (convolutional neural networks), [13] and the use of 3D information, [14-19].

Using visual information and ultrasonic sensors to traverse doors was an approach used in [7]. The goal was to traverse an open door with a certain opening angle using a B21 mobile robot equipped with a CCD camera sensor and 24 sonar sensors. The door traverse was divided into two sub-tasks, the door identification and the door crossing. The door identification which was the sub-task of interest for this work, used a vertical Sobel filter applied to the grey-scaled image. If there was a column wider than 35 pixels in the filtered image it would mean that image contained a door. The sonar sensors were used when the robot approached the door at a distance of 1 meter to confirm if it was a door or not.

The use of visual information and sonar sensors was not restricted to [7]. In [8], a 2D camera is used for long-range door detection and once detected, sonars are used to perform door classification (open doors). For the door detection, Sobel filters are used to detect vertical stripes in the image, which are then marked by applying to the resultant image an edge closure, composed by a generalised dilation followed by a generalised erosion. The location of possible doors is based on the expected dimensions of the doors and the direction and distance from the walls to the robot. With this information, the robot guides towards the suspected door to detect if it is an open door using the sonars sensors.

A laser-based approach for door and handle identification in indoor environments is used in [10]. In this work, a mobile manipulation platform $P R 2$ robot with a Hokuyo UTM-30 laser sensor. The core of this approach, is to obtain 3D point clouds from the laser sensor and segment the parts of interest for door detection using robust geometric estimators and intensity distribution variations in the scan. Colour information (2D image), is not used in any part of this approach algorithm since it is highly influenced by light variations. The disadvantage of this method is that it is tested in a controlled environment. This approach was developed for detecting doors and handles using the requirements imposed by ADA (American Disability Act).

In [11], an integrated solution to recognize a door and its knob in an office environment using a humanoid platform is proposed. The goal is for the humanoid to recognize a closed-door and its knob, open the same door and pass through it. To recognize a door they match the features of the input image with the features of a reference image using the STAR Detector [20] as the feature extractor and an on-line randomised tree classifier to match the feature points. If the door is in the scene, the matched 
feature 3D points are computed and used so that the robot walks towards the door.

The use of colour and shape information can be sufficient for identifying features to efficiently detect doors. The approach in [9] used two neural networks classifiers for recognizing specific components of the door. One was trained for detecting the top, left and the right bar of the door and the other was trained for detecting the corners of the door. A door is detected if at least 3 of these components are detected and have the proper geometric configuration.

In [13], a method is implemented for detecting doors/ cabinets and its knobs for robotic grasping using a 3D Kinect camera. It uses CNN to recognize, detect and segment the ROI (region of interest) in the image. The CNN used was the YOLO Detection System trained with 510 images of doors and 420 of cabinets from the ImageNet dataset. After obtaining the ROI, the depth information from the 3D camera is used to obtain handle point clouds for robot grasping.

Like the previous approach, in [14], a Kinect sensor is used for door detecting but, this method uses only depth information. The camera sometimes produces missing points in the depth image, and the algorithm is based in the largest cluster of missing pixels in the depth image. The total number of holes indicates the status of the door, (open or semi-open). The main advantage of this method is that it works with low-resolution depth images.

There are methods developed under a 6D-space framework, like [15], that use both colour (RGB) and geometric information (XYZ) for door detection. For detecting open doors they detect rectangular point cloud data gaps in the wall planes. The detection of closed doors is based in the discontinuities in the colour domain and in the depth dimension. It also does door classification between open and closed doors. The improved version of this algorithm, [17], can even distinguish semi-open doors using the set of points next to the door to calculate the opening angle. Another improvement in [17] was in the dataset, which is larger in size, complexity and variety.

In [16], a method is proposed that uses 3D information for door detection without using a dependent training-set detection algorithm. Initially, the point cloud containing all the scene, including the door, is prepossessed using a voxel-grid filter to reduce its density and its normal vectors are calculated. A region growing algorithm based on the pre-calculated normals is used to separate the door plane from the rest of the point cloud and after that, feature extraction is used to get the edges of the door and the doorknob.

To detect doors, 3D cameras or sonar sensors are not required, a simple RGB camera can do the job as in [12], focusing on real-time, low-cost and low-power systems.
This work used the Adaboost algorithm to combine multiple weak classifiers into a strong classifier. The weak classifiers were based in features such as detecting pairs of vertical lines, detecting the concavity between the wall and the doorframe, texture and colour and others. They built a dataset with 309 door RGB images, 100 for training their algorithm and the rest for testing.

In [18], an approach that combines the information of neural networks with efficient point cloud processing for door and handle detection is proposed. The goal was to enable a robot (Toyota Human Support Robot) to open doors autonomously, regardless the door form or kinematic model. To detect doors and door handles the YOLO algorithm, trained in a custom dataset, was used. This dataset was built by annotating images from Open Images Dataset that contained the classes "door" and "handle". The 3D information was used to determine the door plane normal and the handle position by computing the 6-D pose in real-time, using Regions of interest (ROIs) segmentation.

An Al-enabled framework was proposed in [19] through a Human Support Robot (Toyota HSR) for COVID-19-like pandemic situations. The role of the robot was to disinfect the door handles. It used a lightweight deep-learning (CNN Object detection) technique for the classification of the door handle image space. The robot used was also equipped with a RGBD camera and it used a NVIDIA Jetson TK1 to execute the door-handle detection. The base framework used for this task was the YOLO V 3 neural network, which was trained to detect and classify door handles. The $3 \mathrm{D}$ information is used to convert the detected bounding box of the door handle in the 3D space.

Table 1 summarises the previous approaches and related work to detect and classify doors in indoor spaces, categorising each method studied. Although most of the approaches just do door detection and not classification of their state, as we did in this work, they have the same goal, to provide the robot with the necessary information to move between rooms, and that is the reason why we included them in this paper. The first column states whether the method uses 3D information or not. The following 3 columns state the applicability of the method (closed, open or semi-open doors). The last column focus on whether the method works in real-time or not, based on the experimental results of each method. Four of the methods do not present information regarding their speed and are marked with a "-".

\section{Problem definition}

Mobile robots nowadays are used for multiple tasks and purposes in several indoor environments as security guard robots, tour guide robots, vacuum cleaners and 
Table 1 Related work comparison (door detection)

\begin{tabular}{|c|c|c|c|c|c|}
\hline Method & $3 \mathrm{D}$ & $\begin{array}{l}\text { Closed } \\
\text { doors }\end{array}$ & Open doors & $\begin{array}{l}\text { Semi- } \\
\text { open } \\
\text { doors }\end{array}$ & Real-time \\
\hline $\begin{array}{l}\text { Monasterio } \\
\text { [7] }\end{array}$ & $x$ & $x$ & $\checkmark$ & $x$ & - \\
\hline Stoeter [8] & $x$ & $x$ & $\checkmark$ & $x$ & $\checkmark$ \\
\hline Cicirelli [9] & $x$ & $\checkmark$ & $\times$ & $\checkmark$ & $x$ \\
\hline Rusu [10] & $\checkmark$ & $\checkmark$ & $\checkmark$ & $\checkmark$ & $\checkmark$ \\
\hline Kwak [11] & $x$ & $\checkmark$ & $x$ & $x$ & $\checkmark$ \\
\hline Chen, [12] & $x$ & $\checkmark$ & $x$ & $x$ & $\checkmark$ \\
\hline Llopart [13] & $\checkmark$ & $\checkmark$ & $\checkmark$ & $\checkmark$ & $\checkmark$ \\
\hline Yuan [14] & $\checkmark$ & $x$ & $\checkmark$ & $\checkmark$ & - \\
\hline $\begin{array}{l}\text { Quintana } \\
\text { [15] }\end{array}$ & $\checkmark$ & $\checkmark$ & $\checkmark$ & $x$ & - \\
\hline Borgsen [16] & $\checkmark$ & $\checkmark$ & $x$ & $\times$ & $\times$ \\
\hline $\begin{array}{l}\text { Quintana } \\
\text { [17] }\end{array}$ & $\checkmark$ & $\checkmark$ & $\checkmark$ & $\checkmark$ & - \\
\hline $\begin{array}{l}\text { Arduengo } \\
\text { [18] }\end{array}$ & $\checkmark$ & $\checkmark$ & $x$ & $x$ & $\checkmark$ \\
\hline $\begin{array}{l}\text { Ramalingam } \\
\text { [19] }\end{array}$ & $\checkmark$ & $\checkmark$ & $x$ & $x$ & $\checkmark$ \\
\hline Ours & $\checkmark$ & $\checkmark$ & $\checkmark$ & $\checkmark$ & $\checkmark$ \\
\hline
\end{tabular}

others. Usually, in these environments, the robot has to navigate safely between rooms and the biggest obstacles are the doors. The mobile system normally must be able to detect the door in the scene to move to another room. In more complex situations, the robot has not only to detect the door but also has to classify its state to decide its next move.
Door detection is used in situations where the door opening is stationary and in situations where the door could either be totally open or closed.

Door state classification is useful in difficult situations where the door, in addition to open and closed, can also be semi-open. We decide to work with door state classification because it can be used by the robot to solve more complex tasks.

In this work, we focus only in the door detection and state classification using computer vision algorithms and methods without concerning the after processes and the action that the robot will take according to the opening of the door. We propose that if the door state is classified as closed, the robot must call a human for help. If it is open the robot can simply go through it and if it is semi-open, the robot can either get around it or try to open it simply by gently pushing it.

The door state can be classified as open, closed and semi-open depending on the door opening angle (angle between the door and the wall where the door is inserted). Doors with opening angles between 0 and $10^{\circ}$ are closed, with opening angles between 10 and $70^{\circ}$ are considered semi-open and with opening angles higher than $70^{\circ}$ are considered open. We also take into account the case of doors with negative angles. This classification is done in the same way as the previous one but with the corresponding negative angles. Figure 1 treats the door opening angles thresholds from a perspective seen from above.

Although the door opening angle is the most important classification factor it is not the only one. We also have in consideration the position of the viewer in relation to the door as a classification factor. For example, the door has an opening angle of $75^{\circ}$ but the position
Fig. 1 Opening angles thresholds for closed, semi-open and open doors

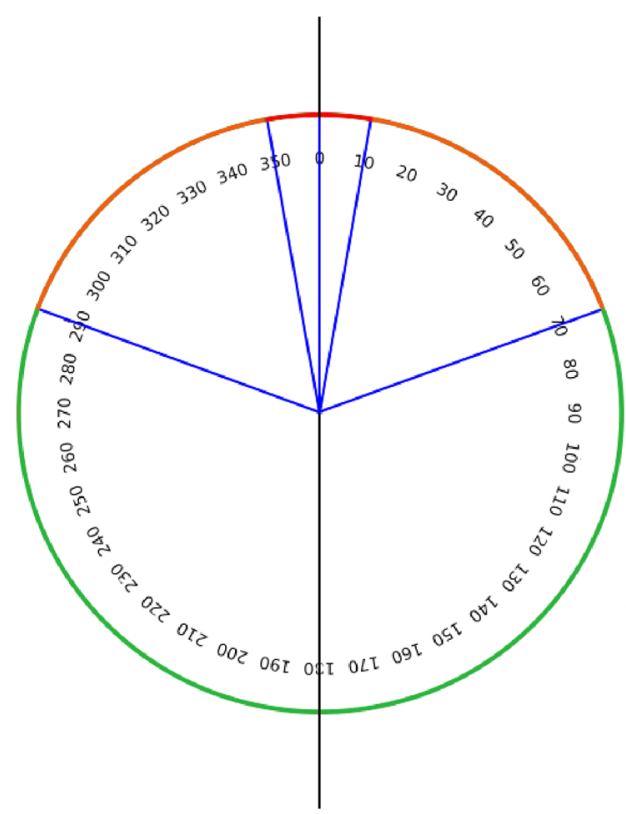

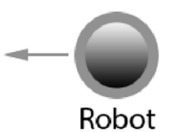

Door

Wall

Open door threshold Semi-open door threshold Closed door threshold 
Fig. 2 Algorithm of Method $A$ (2D semantic segmentation and 3D object classification)
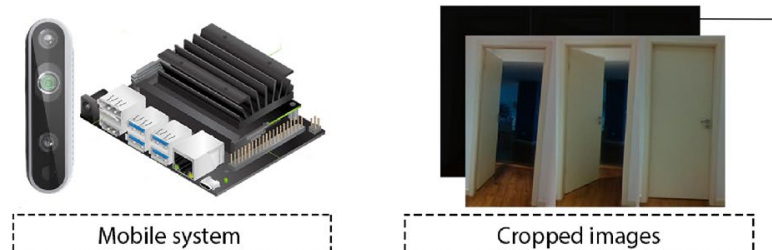

Cropped images

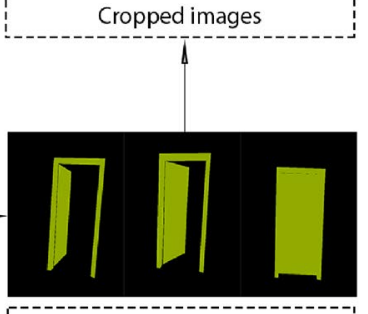

RGB Semantic Segmentation

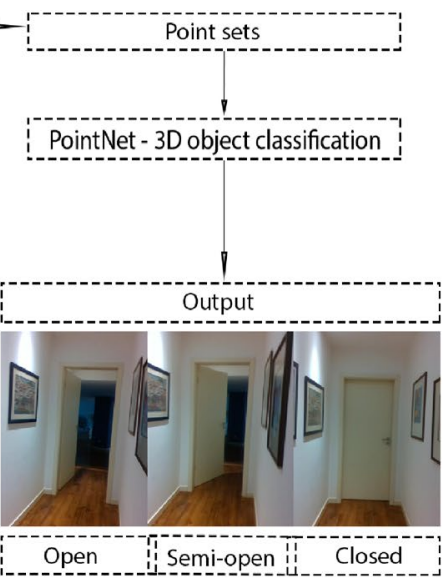

of the robot does not allow it to walk forward and go through the door without the need to get around it. In this case, we considered the door as semi-open, because the robot must get around it to go through it. We decide to do this approach with the objective of making this work applicable to other areas.

The main goal of the robot is to know if there is enough space to move to the next division, however, with our approach, we can further extend the applicability of this method to other problems (navigation for visually impaired people) by using the aperture angle information. The opening angle degree allows to know if a robot with different structure and dimensions such as, the Turtlebot, the Savioke, the Cobalt and others, can pass through the free space of the door opening and move to the other division.

It is also possible to change the opening angles thresholds for closed, semi-open and open doors based on the robot dimensions by changing the labels of the door aperture classification dataset.

\section{Proposed method}

We propose three different methods for door state classification.

The first method, presented in Sect. 4.1, uses the combination of 2D semantic segmentation algorithms with the 3D object classification method. The second method, presented in Sect. 4.2, only uses the 3D object classification method. The third method, presented in Sect. 4.3, uses only 2D information with door detection or segmentation followed by a 2D object classification algorithm.

\subsection{Method A-2D semantic segmentation and 3D object classification}

For this method, we use both RGB and depth information for door state classification, using both of our datasets.

After receiving both RGB and depth frames from the Realsense 3D camera we use a semantic segmentation method and draw a bounding box around the biggest area of pixels of the "door" class resulting from the semantic segmentation. The depth channel is aligned with the RGB channel. The depth image is cropped according to the bounding box of the RGB image, resulting in a depth image with only the door. Using the Open3D library [21], we converted the cropped depth image to a grey-scale point cloud. The point cloud goes to the 3D object classification PointNet, [22], trained with our dataset for PointNet, with 3 classes. The PointNet does the inference with the point cloud and returns the result of the classification.

Figure 2 represents the described method.

Regarding the semantic segmentation algorithms we use the FastFCN Rethinking Dilated Convolution in the Backbone for Semantic Segmentation, [23] and the Fully Convolutional HarDNet which was based in the HarDNet: A Low Memory Traffic Network, [24]. The FastFCN was used because its test score was in the first three best global ranks for semantic segmentation in the ADE2OK dataset. We also tried to implement the EncNet, [25] which is the network that the FastFCN is based on, but the implementation provided could only work in multi-GPU machines. The ADE2OK dataset is very important for door semantic segmentation since the "door" class is labelled and it has indoor images with doors. If a semantic segmentation method performs well in this dataset it will also perform well in ours. We also used the FC-HarDNet because it had the best global rank metric value for real-time semantic segmentation in the Cityscapes dataset. We used it because it was faster than the previous method and we were pursuing a real-time door state classification method. 
Fig. 3 Algorithm of Method $B$ (only 3D object classification)
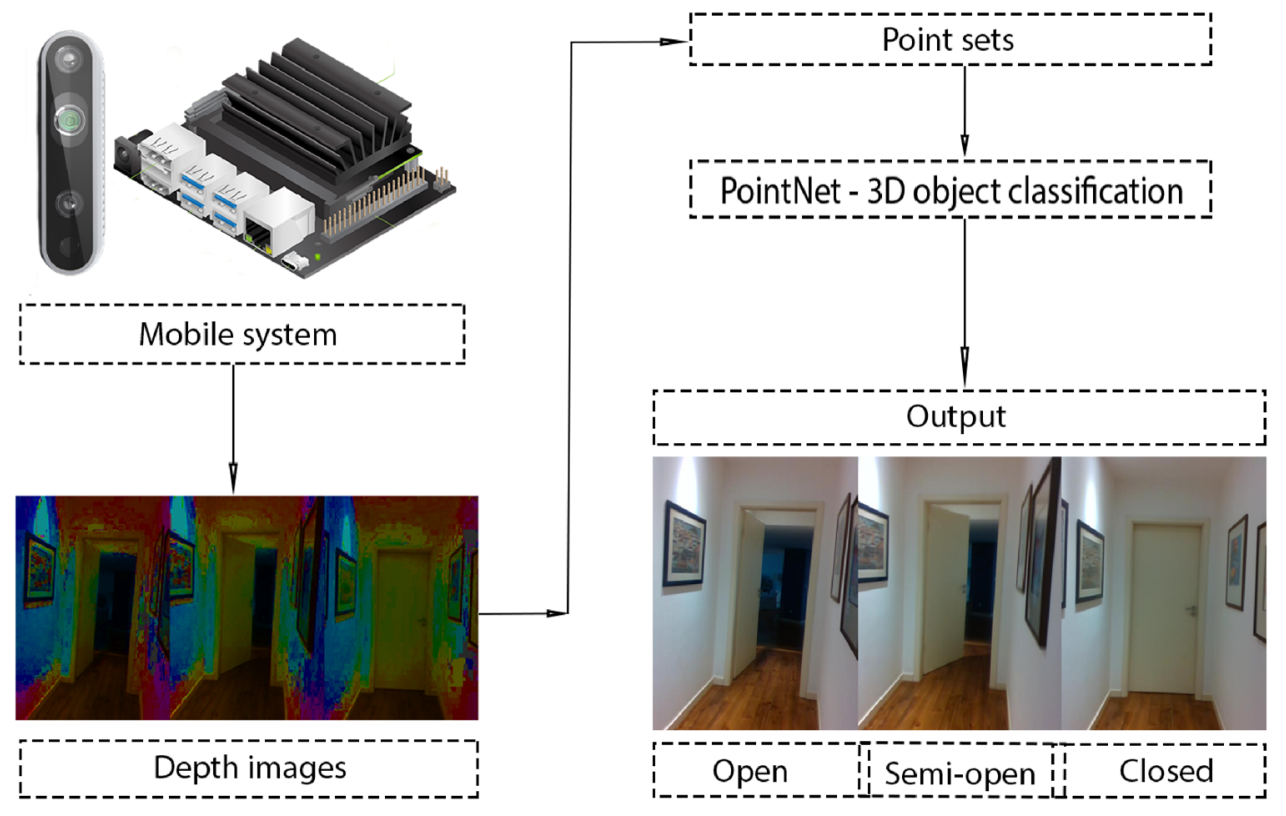

As the 3D object classification method, we used the PointNet. This method accepts unordered point sets and classifies them according to their 3D shape. We used the provided repository in [22] and changed the default dataset which was the ShapeNet to our dataset adjusting the data loader and the number of classes accordingly.

The difference between the methods of this family is in the $2 \mathrm{D}$ semantic segmentation algorithm used (FastFCN and $F(-H a r D N e t)$. These methods are compared later in Sect. 6.

\subsection{Method B-3D object classification}

For this method, we only used the 3D object classification method PointNet. Instead of receiving both RGB and depth data, we use only the depth data. The depth data is converted to a point cloud using the Open $3 D$ library and then converted to a point set. These point sets are the input of the PointNet. Unlike the previous methods, $A$, this method uses the entire point cloud without cutting it because we do not have the bounding box of the door. Although the point cloud is bigger, because it is not cropped, the number of points that enter the PointNet is the same. This happens because the PointNet has a parameter, "number of points", which we will denote by $K$, that defines the number of points of the input point set that will be randomly selected and classified. This method's algorithm is visually represented in Fig. 3.

This method is faster than the previous one in terms of frames per second because it does not use semantic segmentation algorithms and uses the same 3D object classification algorithm.
One parameter we can adjust in the method is if the point cloud undergoes uniform downsampling or not. The $K$ parameter makes PointNet randomly select that number of points and if we have a big point set we might get a set that does not represent uniformly the depth data. We use the uniform downsampling algorithm from Open $3 D$ in the original point cloud, with approximately 300000 points, to get a downsampled version of the same 10 times smaller. The default value of $K$ was 2500 which was too small for our point sets (300 000 or 30000$)$. This number was increased to 10000 and could not be further increased because of the small GPU memory of the mobile system.

\subsection{Method C-2D door detection and 2D door state classification}

For this method, we only used 2D RGB information. We tested object detection and segmentation algorithms because both of these types of methods can be used to obtain the door location in the input image. In Sect. 6, we further detail on the advantages and differences between using door detection and door segmentation. After obtaining the door location, the image is cropped accordingly, and we use a 2D image classification method to obtain the door state classification.

Regarding the object detection and segmentation methods we used DetectNet [26], SegNet [27], and BiSeNet [28]. The DetectNet and SegNet were used because they are, respectively, the neural networks primitives for object detection and semantic segmentation, provided by the jetson-inference repository. This repository is the Hello $\mathrm{Al}$ guide for deploying deep-learning inference networks into NVIDIA Jetson systems. These networks are based 
Fig. 4 Algorithm of Method C (2D door detection and $2 \mathrm{D}$ door classification)
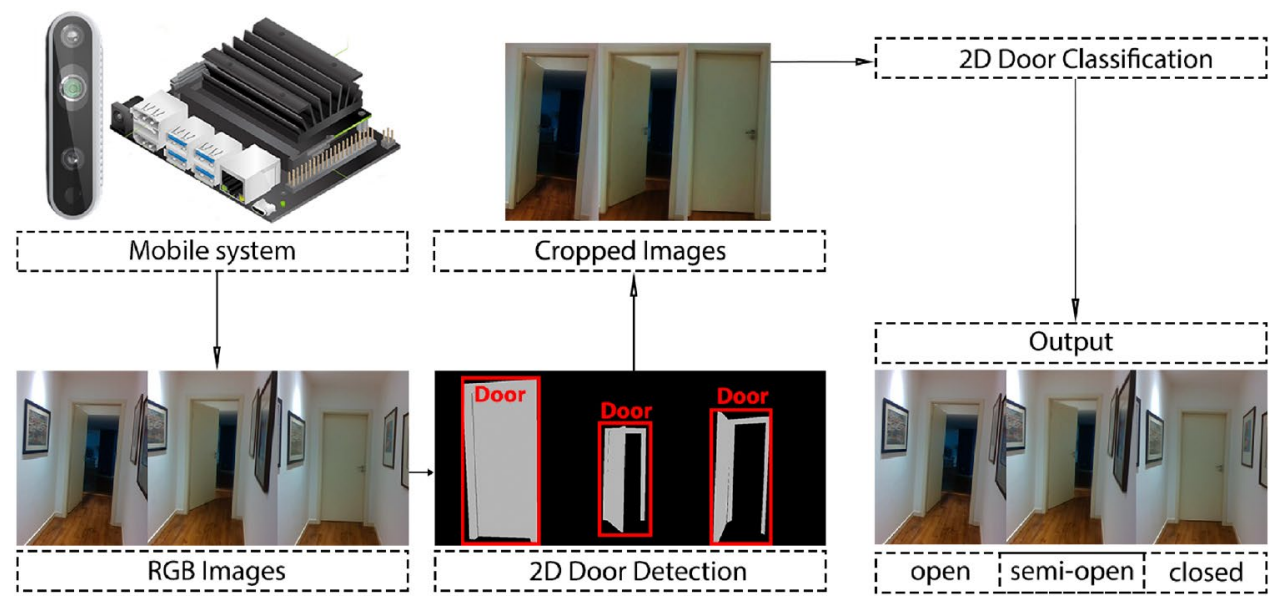

in NVIDIA TensorRT which improves inference speed and power efficiency using graph optimisations, kernel fusions, and FP16/INT8 precision. The BiSeNet is a real-time semantic segmentation method and we used it since it is the fastest model with a mloU precision superior to $74.7 \%$ in the Cityscapes dataset. The DetectNet and SegNet were already provided in tensorRT format by jetson-inference. BiSeNet, on the other hand, is provided as a torch model, and we later converted it into a TensorRT model. To convert the model, we used the torch onnx export method to convert from torch model to a onnx model. After that, we used the onnx tool, onnx-TensorRT tool, to convert onnx to TensorRT. Onnx stands for Open Neural Network Exchange, and it is an open format built to represent machine learning models. We did not use the semantic segmentation algorithms of method $A$ [6], since the first one: [23], was not compatible with Jetson Nano and the other algorithm: [24], was not capable of detecting all of the doors in the test set of the previous work dataset.

Regarding the object classification neural networks, we used the AlexNet and GoogleNet networks. We used these networks since they were also provided in the jetson-inference as the image classification primitives networks and they were already in the TensorRT format (Fig. 4).

One of the advantages of this method is that it does not require 3D information, thus, it does not force the system to have a 3D camera to perform door detection and state classification. Another advantage is that it provides the door detection as method $A$ provides, unlike method $B$.

\section{Datasets}

In our previous work, [6], we built two datasets, one for the 3D object classification algorithm, PointNet and the other for the semantic segmentation algorithms.
The first dataset is constituted by RGB images and corresponding depth images both with size of $480 \times 640$ pixels. In total, this dataset has 1206 door images, where 468 of the images are of closed doors, 588 of open doors, and 150 of semi-open doors. For the test and validation set, we used 20 samples of each class giving a total of 60 samples for test and 60 for validation. We used the remaining samples of each class to build the training set, representing a total of 1086 images.

The second dataset was built by annotating images from the previous dataset using the Computer Vision Annotation Tool (CVAT) [29]. This dataset is constituted by 240 grey-scaled images with the size $480 \times 640$ pixels, and it was divided into train and test sets with 200 and 40 samples respectively.

The aforementioned datasets have several disadvantages. First, the datasets are not balanced, having almost 4 times more opened door images than semi-opened door images. Second, the validation and test sets of these datasets are small, since we only have 20 samples per class on each of these sets. Finally, the semantic segmentation dataset is very small and does not have a validation set.

Due to the mentioned disadvantages, we built a new dataset that solved all of the aforementioned problems, named DeepDoors Version 2.0 Dataset. This dataset is constituted by 3 parts, a 2D and 3D image classification part, a semantic segmentation part and an object detection part. For the first two parts, we used the previous work dataset and improve it, by collecting more data and annotating more images. The third part was built by annotating images with the CVAT from the image classification part.

This dataset was built using a portable system constituted by a Raspberry Pi 3 B + powered by a power-bank with a 3D Realsense Camera, model D435. This camera has a horizontal viewing angle $\left(86^{\circ}\right)$ higher than the vertical viewing angle $\left(57^{\circ}\right)$. We rotated the camera $90^{\circ}$ to switch the angles with the purpose of including all the door area 
Table 2 Dataset comparison with previous and related work

\begin{tabular}{lccl}
\hline DataSet & 3D & RGB & $\begin{array}{l}\text { Number } \\
\text { of sam- } \\
\text { ples }\end{array}$ \\
\hline Chen [12] & $\times$ & $\checkmark$ & 309 \\
Llopart [13] & $\times$ & $\checkmark$ & 510 \\
Rusu [10] & $\checkmark$ & $\times$ & 50 \\
Quintana [17] & $\checkmark$ & $\times$ & 35 \\
Arduengo [18] & $\times$ & $\checkmark$ & 1213 \\
Ramalingam [19] & $\times$ & $\checkmark$ & 4500 \\
Crivellaro [30] & $\checkmark$ & $\checkmark$ & 3000 \\
Previous work [6] & $\checkmark$ & $\checkmark$ & 1206 \\
DeepDoors Version 2.0 & $\checkmark$ & $\checkmark$ & $\mathbf{3 0 0 0}$ \\
\hline
\end{tabular}

The significance of [bold] was to highlight our number of samples in the dataset from the others datasets

in the image. The camera was placed $135 \mathrm{~cm}$ above the floor.

We captured several images of doors and their surroundings with different textures and sizes. Some images have obstacles that obstruct and hide part of the door such as, chairs, tables, furniture and even persons. The goal was to create a more generic and realistic real-world dataset. We also changed the pose to get different perspectives on the same door. The images captured are from our university, public places and people's houses. All of the images were manually captured using a portable system with a Jetson Nano and manually annotated using the CVAT, [29], for door segmentation, detection and state classification.

\subsection{Deepdoors version 2.0-door state classification}

This dataset is constituted by RGB images and corresponding depth images with a size equal to $480 \times 640$ pixels. The depth images are in grey-scale with pixels values between 0 and 255 and we used a depth scale equal to $1 / 16$. The depth in meters is equal to depth scale * pixel value, for example, if the pixel value is equal to 32 it means that that pixel is $2 \mathrm{~m}$ away from the viewer $(1 / 16 * 32=2)$.

In total, this dataset has 3000 door images, 1000 samples for each class: open, closed and semi-open doors. This dataset was randomly split into: 300 samples for validation, 300 for testing and 2400 for training. Figure 5, presents a few images from our dataset.
Fig. 5 Sample images from DeepDoors Version 2.0 dataset

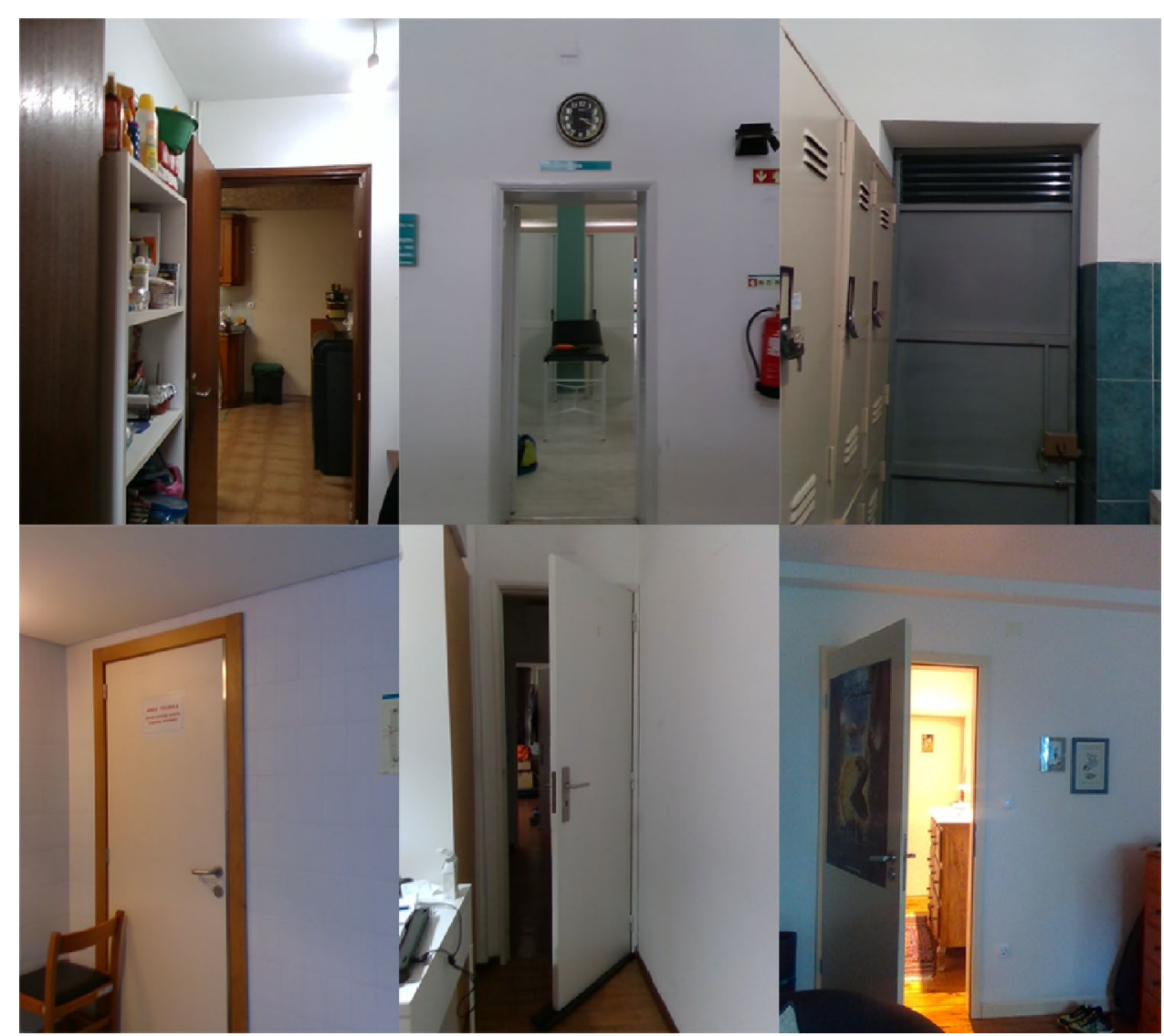


We compared this dataset with our previous and the related work datasets in terms of numbers of samples and $3 \mathrm{D} / \mathrm{RGB}$ coverage in Table 2. From this table, it is possible to see that our dataset has more samples than the majority of the related-work datasets and it has both RGB and depth information.

In $[12,13]$ and $[18]$, the datasets built have a substantial number of samples but do not have 3D information, which incapacitates 3D-based door classification and detection methods that rely on 3D datasets. In [18], 3D information for door pose and handle detection is used. However, this method does not use a 3D dataset.

The largest dataset in terms of number of samples is the one built in [19]. However, this dataset was built by collecting images through Bing image search and using the dataset developed in [18]. It was built for door handle detection sorted into three classes, circle type, lever type and bar type handles. This dataset couldn't be used entirely for door detection, as several images contain only the door handle zoomed with almost no information of the door itself.

The datasets developed in [10] and [17] are constituted by point clouds (3D information) but have a reduced number of samples, and the data is from a controlled environment (one only building).

We also compared our dataset with datasets developed for substantial different purposes and goals such as, the DOOR dataset built in [30]. The authors proposed a method for detecting the 3D pose of a known object using grayscale images without the need of depth sensors. The dataset has images of one non-textured door being opened and closed by a user. It contains 3000 samples that were created by using the CAD model of the door and, the ground-truth pose for all the sequences. The images were registered using the $A R U C O$ marker tracking tool. Although it is used for 3D pose estimation, this dataset can be used for door state classification. The biggest disadvantage of this dataset is that, it contains only one door, consequently it is not good for model generalisation.

One advantage of our dataset, beyond the number of samples is its environment diversification. Our dataset has images from several environments and different places, such as, university facilities, house images, countryside houses with nature. Moreover, it is also constituted by several blur images to simulate a real world application, as well as obstacles that cover a substantial part of the door.

\subsection{Deepdoors version 2.0 - semantic segmentation}

To build a semantic segmentation dataset we used the Computer Vision Annotation Tool (CVAT) [29] as we did in our previous work. This dataset was built by annotating images from the image classification strand of the DeepDoors Version 2.0 dataset. Using the polygons mode of the CVAT, we drew rectangles around the doors and door frames in each image.

This dataset has 3000 grey-scaled images with the size of $480 \times 640$ pixels. The splits were randomly created before annotating the images. Thus, the train set contained 2400 images, the validation set contained 300 images and the test set contained 300 images.

As we are just concerned with the doors and door frames, we only used two classes in this dataset. The pixel value is 1 if it corresponds to a door or door frame, and is 2 if it does not.

\subsection{Deepdoors version 2.0-Object Detection}

This last part, was built for training and testing the object detection method DetectNet [26]. As for the semantic segmentation part dataset, we used the CVAT to annotate the images. The object detection and semantic segmentation have exactly the same number of samples, having as only difference the annotation. For annotating the images for object detection we used bounding boxes around the door and door-frame instead of using the polygon mode as we did in the semantic segmentation part. The annotations consist of the bounding boxes width minimum and maximum and height minimum and maximum.

This dataset only has 2 classes: door, which corresponds to a door and doorframe, and not door, which corresponds to all the other objects that are not doors. The splits for test, training and validation sets were exactly the same as the door segmentation strand, with the purpose to compare segmentation and detection methods trained in the same dataset and tested in the same test split.

The DeepDoors Version 2.0 dataset is divided in the 3 strands, image classification (2D and 3D), semantic segmentation and object detection, and is freely available online. ${ }^{1}$.

\section{Experiments and discussion}

We compared our methods against each other in real-time scenarios. We did not compare with the methods of the related work because their focus was in door detection while ours is in door state classification.

All the image classification, semantic segmentation and object detection models were trained in a machine with $16 \mathrm{~GB}$ of RAM memory, a 256GB SDD disk, an AMD Ryzen 72700 processor with 16 Threads and a GeForce

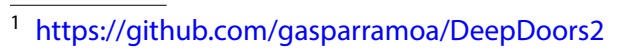


GTX 1080Ti graphics card with $12 \mathrm{~GB}$. The mobile system where the speed tests were conducted is composed by a Jetson Nano in $10 \mathrm{~W}$ mode with a Realsense 3D camera D435 with no fan.

It is important to mention that we did not change any of the algorithms used in our methods as the PointNet, FastFCN, FC-HarDNet, SegNet, AlexNet, GoogleNet and DetectNet with the exception of the BiSeNet. As it was aforementioned, the BiSeNet torch model was converted to a TensorRT model with the goal to improve the inference time. In the desktop machine, the BiSeNet torch model mean inference speed is 35 FPS while on the TensorRT model version, it was increased to $52 \mathrm{FPS}$. By converting it to a TensorRT model we gained 17 FPS.

For all the other algorithms we only changed the data loaders and did the necessary configurations to work with our data sets.

In our previous work, we tested the FastFCN and FCHarDNet semantic segmentation algorithms for Method $A$, we tested one important parameter of the PointNet for Method $B$ and then we compared both of these methods in terms of test accuracy and inference time. In this paper, we tested different computer vision algorithms that could be used in Method C, from 2D image classification, 2D object detection and 2D semantic segmentation algorithms. We also made a final comparison between all of the proposed 3 methods, $A, B$ and $C$.

\subsection{Method C}

Method C uses a 2D object detection or semantic segmentation network to detect the door to provide the necessary door information (cropped by a bounding box) to the 2D image classification network.

\subsection{Method C-Detection}

For the door detection we tested one object detection network, DetectNet and two semantic segmentation networks, SegNet and BiSeNet.

The DetectNet was trained in DIGITS (NVIDIA) which is a GUI for training neural networks. We used our previous work dataset since we compared these results later in this paper with Method $A$ and $B$ and it would not be fair to compare the methods with different training and test sets. The difference between the datasets was in the annotation. Instead of 240 grey-scaled images with 2 pixel values that represent door and no-door classes, we used the YOLO annotation for object detection. Seg$\mathrm{Net}$ and BiSeNet were trained with the same dataset. To compare the object detection model with the two semantic segmentation models we counted the True
Table 3 Evaluation and Comparison of DetecNet, SegNet and BiSeNet on Door Detection/Segmentation in terms of number of True Positives(TP), number of False Positives(FP), mean inference and post inference in seconds and total inference time in Jetson Nano in FPS (frames per second)

\begin{tabular}{llllll}
\hline Method & TP & FP & $\begin{array}{l}\text { Mean } \\
\text { Inference(s) }\end{array}$ & $\begin{array}{l}\text { Post } \\
\text { Inference(s) }\end{array}$ & $\begin{array}{l}\text { Total } \\
\text { Inference }\end{array}$ \\
\hline DetectNet & $28 / 40$ & $10 / 40$ & 0.130 & 0 & 7 FPS \\
SegNet & $40 / 40$ & $40 / 40$ & 0.400 & 0.006 & 2 FPS \\
BiSeNet & $38 / 40$ & $04 / 40$ & 0.400 & 0.006 & 2 FPS \\
\hline
\end{tabular}

Positives and False Positives. We used 40 door images from the previous test set dataset and we used 40 random images with no doors from the COCO dataset, [31] to represent the negative cases. In DetectNet we used a threshold coverage value equal to 0.7. In BiSeNet and SegNet we calculated the biggest door/doorframe area in the semantic segmentation output using Dilation followed by Erosion filters. We used a threshold pixel area equal to 30 thousand pixels for the semantic segmentation networks.

For each method, we measured its inference time and post inference time (just for the semantic segmentation approaches) in seconds in Jetson Nano. The total time represents the time, in frame per second, that it takes to provide the cropped RGB image to the image classification network in Method C. Table 3 presents the evaluation and comparison of DetecNet, SegNet and BiSeNet on Door Detection/Segmentation in terms of the number of True Positives, False Positives, the mean inference time, post inference and total time in Jetson Nano.

The SegNet model did not learn to segment doors, regardless of the input image, this model always returned the same output, an image with just door/ doorframe pixels. That is the reason why it detected all true positive cases and got all the possible false-positive cases. Summing up, SegNet from the jetson-inference is not yet well implemented in this repository and so, it is not the most suitable door detection or segmentation network for Method $C$.

DetectNet works at 7 FPS in Jetson Nano and outputs the bounding box coordinates of the detected door, and that is why its post inference time is 0 seconds. If we compare DetectNet with BiSeNet in terms of inference speed, it is clear that DetectNet is faster but it is not able to detect as many doors as BiSeNet. In this test, BiSeNet only failed to segment 2 out of 40 doors and just detected 4 doors out of 40 images with no doors. DetectNet detected 10 fewer doors than BiSeNet and it detected 6 more doors in the negative images. In view of the above, we opted to use BiSeNet for door segmentation 
Table 4 Comparison of image classification networks for Method C in DIGITS in terms of the neural network used, training set batch size, input images size, accuracy in the test set and inference time on Jetson Nano in frames per second

\begin{tabular}{lllll}
\hline Neural Network & Batch size train set & Input size & Accuracy Test & Jetson inference \\
\hline AlexNet & 128(default) & $480 \times 640$ & 56.67 & $55 \mathrm{FPS}$ \\
GoogleNet & 32(default) & $480 \times 640$ & 36.67 & $65 \mathrm{FPS}$ \\
AlexNet & 128 (default) & $227 \times 227$ & 95.00 & $55 \mathrm{FPS}$ \\
AlexNet & 6 & $227 \times 227$ & $\mathbf{9 8 . 3 3}$ & $55 \mathrm{FPS}$ \\
GoogleNet & 32 (default) & $224 \times 224$ & 91.67 & $65 \mathrm{FPS}$ \\
GoogleNet & 6 & $224 \times 224$ & 93.33 & $65 \mathrm{FPS}$ \\
\hline
\end{tabular}

The significance of [bold] is the highest accuracy from all the neural networks used
Table 5 Methods comparison in terms of door segmentation, state classification and total time in frames per second. For the semantic segmentation network, we present the mean test intersection over

\begin{tabular}{llllllll}
\hline Method & Seg. network & Seg. loU & Seg. time(s) & Class. network & Class. acc. & Class. time(s) & Total Time \\
\hline A & FC-HardNet & 0.418 & 0.131 & PointNet & 0.494 & 0.111 & 3 FPS \\
B & $\times$ & $\times$ & $\times$ & PointNet & 0.433 & 0.111 & 6 FPS \\
C & BiSeNet & 0.822 & 0.412 & AlexNet & 0.983 & 0.019 & $1-2$ FPS \\
\hline
\end{tabular}

union and the inference time in seconds. In terms of door state classification, we show the network used, the mean test accuracy and the inference time in seconds in Method C, since the results in terms of number doors detected were the best out of the 3 algorithms.

\subsection{Method C-Classification}

We tested two image classification networks, the GoogleNet and the AlexNet. We used our previous work dataset for the same reasons mentioned in the previous test. We cropped the images of the dataset according to the door bounding boxes to simulate the output of the object detection or semantic segmentation method. These images were resized to $480 \times 640$ since the images have different dimensions due to different crops of bounding boxes and the image classification methods required a fixed input size.

We trained both GoogleNet and AlexNet networks for 100 epochs using a learning rate equal to $2 \times 10^{-2}$ with a step-down policy and with a batch size of 32 . The training was done in DIGITS (NVIDIA). Initially, we tested both networks with the previous parameters and the test accuracy was $56.67 \%$ for AlexNet and $36.67 \%$ for GoogleNet.

The AlexNet model uses data augmentation by cropping the original image into a $227 \times 227$ image which could lead to bad results since it just represents $16.8 \%$ of the original image. To solve this problem we resized the images from $480 \times 640$ to $227 \times 227$. The other training parameters remained the same. The test accuracy was $95 \%$. After this test, we changed the batch size from 128 to 6 . As the batch size was smaller, the number of iterations per epoch increased, and for its consequent, the training time also increased, but resulted in a test accuracy of $98.33 \%$
The GoogleNet model also uses data augmentation by cropping the input images but instead of $227 \times 227$ images it uses $224 \times 224$ images. We also resized the original training images to $224 \times 224$ images and kept the other training parameters. The test accuracy was $91.67 \%$. The default GoogleNet batch size is 32, and as we did for the AlexNet, we reduce it to 6 . The highest test accuracy of all epochs after this modification the test accuracy in GoogleNet was $93.33 \%$.

Table 4 represents the previous experiments in GoogleNet and AlexNet. In view of the above we conclude that the more appropriate image classification network to use in Method C is the AlexNet since it got the highest test accuracy running in real-time on low powered devices.

\subsection{Method A vs Method B vs Method C}

Method A and B use the 3D image classification, PointNet for door state classification while Method $C$ uses the 2D image classification AlexNet. Method A and C use 2D semantic segmentation while Method $B$ is more focused in real-time and just does $2 \mathrm{D}$ door state classification. We compared all three methods in terms of door segmentation, door state classification and total inference time in Jetson Nano. For door segmentation, we used the Intersection over Union as the evaluation metric in the test set and we also compared the mean inference time in seconds. For door state classification, we compared the mean accuracy in the test set and the mean inference time in seconds. All of the methods and its algorithms were trained and tested in the previous work dataset. Table 5 represents this comparison. 
Analysing the results of Table 5 we came to the conclusion that Method $C$ is the most suitable for door detection and state classification in low powered devices. In terms of door segmentation BiSeNet from Method C has the highest test intersection over union, 0.822 while FC-HardNet from Method A only has 0.418 . In terms of door state classification, Method $C$ stands out with the highest test accuracy $(98.3 \%)$ and with the fastest inference time $(0.019 \mathrm{~s})$ when compared to the 3D image classification PointNet from Method $A$ and $B$. The big drawback of the Method $C$ is the total inference time. This method can only work at 1 to 2 FPS in Jetson Nano, which is slow when compared with the other methods. However, the accuracy it gains in door classification and the addition of door detection with good loU test values counterbalance the processing time, making it the best methodology for door detection and state classification in this context.

\subsection{Comparing with others}

We did not compare our methods with the ones from related works because those works focused on door detection, while we did door state classification. The Llopart method, [13], works in every class of door as it can be seen in table 1, but it does not actually do door state classification since his method cannot recognize the difference between doors, it just detects them. Quintana's method, [17], is the only method that does door state classification implicitly by differentiating closed doors from open and semi-open doors using the opening angle to differentiate open from semi-open doors. Their dataset ( 35 point clouds) is not as complete as ours (3000 point clouds) and their method does not work in real-time, although it presented excellent results in their dataset.

\section{Conclusion}

In this paper, we proposed three different methods for door detection and state classification to improve robot navigation and to provide it with the information to move between rooms. Each method uses different types of information, and all are capable of working in real-rime in low-power computers such as the Jetson Nano from Nvidia. The robot does not have to be connected to the internet because our methods work offline. To compare the developed methods, we used a single board computer equipped with a 3D camera powered by a powerbank. Inference speed and test accuracy was calculated and compared for each method. A novel dataset was also developed with RGB images and their respective point clouds divided into three sections, one for $2 \mathrm{D} / 3 \mathrm{D}$ image classification with images of closed, open and semi-open doors, another for 2D semantic segmentation with annotated doors and corresponding door frames and one for 2D door detection, all properly and manually annotated.

Our work can be used in other areas and applications, as for systems that help visually impaired people navigate in indoor spaces to improve their lifestyle and other systems that use the information of the door state. Due to the fact that we used the door opening degree information, our methods can be tested in different robots with different structure and dimensions, by defining the opening angles thresholds for closed, semi-open and open doors. This work can be compared to future methods using our online published dataset and our accuracy results on Jetson Nano. Since our methods for door detection and state classification use publicly available algorithms, these can be tested in other datasets. Video based datasets can also be used to test our methods in future works considering that videos can be divided in a set of frames.

For future work we propose to adapt our methods to use more current state-of-the-art algorithms for door segmentation and door state classification. In our methods, we used AlexNet and GoogleNet networks in the TensorRT format for 2D door state classification. As future work, it would be interesting to extend the networks tested, considering other more powerful networks such as, Inception V3, [32], and ResNet, [33]. The same also applies for 3D door state classification and 2D door segmentation. We also desire to extend and replicate the methodologies used for object detection and classification to other scenarios and contexts within the robot navigation topic.

Acknowledgements This work is supported by NOVA LINCS (UIDB/04516/2020) with the financial support of FCT-Fundação para a Ciência e a Tecnologia, through national funds.

The manuscript presented is the extended version of [6]. Three new methods of door detection and classification of their degree of opening are proposed and compared. The open-access indoor image database was also substantially enlarged.

\section{Declarations}

Conflict of interest The authors declare that they have no conflict of interest.

Open Access This article is licensed under a Creative Commons Attribution 4.0 International License, which permits use, sharing, adaptation, distribution and reproduction in any medium or format, as long as you give appropriate credit to the original author(s) and the source, provide a link to the Creative Commons licence, and indicate if changes were made. The images or other third party material in this article are included in the article's Creative Commons licence, unless indicated otherwise in a credit line to the material. If material is not included in the article's Creative Commons licence and your intended use is not permitted by statutory regulation or exceeds the permitted use, you will need to obtain permission directly from the copyright holder. To view a copy of this licence, visit http://creativecommons. org/licenses/by/4.0/. 


\section{References}

1. Wickramaarachchi WHC, Chamikara MAP, Ratnayake RACH (2017) Towards implementing efficient autonomous vacuum cleaning systems, In: 2017 IEEE International Conference on Industrial and Information Systems (ICIIS), pp. 1-6

2. Narayanan KL, Kumaran DNM, Rajakumar G, Arshadh H, Dinesh I, Caleb V (2019) Design and fabrication of medicine delivery robots for hospitals. SSRN Electron J. https://doi.org/10.2139/ ssrn.3432156

3. Sadkhan AB (2019) Design and Implement a Robot for Security Purposes, In: 2019 2nd International Conference on Engineering Technology and its Applications (IICETA), pp. 123-126

4. Huang S, Tanioka T, Locsin R, Parker M, Masory O (2011) Functions of a caring robot in nursing, In: 2011 7th International Conference on Natural Language Processing and Knowledge Engineering, pp. 425-429

5. Yamazaki K, Ueda R, Nozawa S, Kojima M, Okada K, Matsumoto K, Ishikawa M, Shimoyama I, Inaba M (2012) Home-assistant Robot for an aging society. Proc IEEE 100(8):2429. https://doi. org/10.1109/JPROC.2012.2200563

6. Ramôa JG, Alexandre LA, Mogo S (2020) Real-Time 3D door detection and classification on a low-power device, In: 2020 IEEE International Conference on Autonomous Robot Systems and Competitions (ICARSC), pp. 96-101

7. Monasterio I, Lazkano E, Rano I, Sierra B (2002) Learning to traverse doors using visual information. Math Comput Simul 60:347. https://doi.org/10.1016/S0378-4754(02)00027-7

8. Stoeter SA, Le Mauff F, Papanikolopoulos NP (2000) Real-time door detection in cluttered environments, In Proceedings of the 2000 IEEE International Symposium on Intelligent Control. Held jointly with the 8th IEEE Mediterranean Conference on Control and Automation (Cat. No.00CH37147), pp. 187-192

9. Cicirelli G, D'Orazio T, Distante A (2003) Target recognition by components for mobile robot navigation. J Exp Theor Artif Intell 15:281. https://doi.org/10.1080/0952813021000039430

10. Rusu RB, Meeussen W, Chitta S, Beetz M (2009) Laser-based perception for door and handle identification, In: 2009 International Conference on Advanced Robotics, pp. 1-8

11. Kwak N, Arisumi H, Yokoi K (2011) Visual recognition of a door and its knob for a humanoid robot, In: 2011 IEEE International Conference on Robotics and Automation, pp. 2079-2084. https://doi.org/10.1109/ICRA.2011.5979608

12. Chen Z, Birchfield ST (2008) Visual detection of lintel-occluded doors from a single image, In: 2008 IEEE Computer Society Conference on Computer Vision and Pattern Recognition Workshops, pp. 1-8. https://doi.org/10.1109/CVPRW.2008.4563142

13. Llopart A, Ravn O, Andersen NA (2017) Door and cabinet recognition using Convolutional Neural Nets and real-time method fusion for handle detection and grasping, In: 2017 3rd International Conference on Control, Automation and Robotics (ICCAR), pp. 144-149. https://doi.org/10.1109/ICCAR.2017.7942676

14. Yuan TH, Hashim FH, Zaki WMDW, Huddin AB (2015) An automated 3D scanning algorithm using depth cameras for door detection, In: 2015 International Electronics Symposium (IES), pp. 58-61. https://doi.org/10.1109/ELECSYM.2015.7380814

15. Quintana B, Prieto SA, Adán A, Bosché F (2016) Door detection in $3 \mathrm{D}$ colored laser scans for autonomous indoor navigation, In: 2016 International Conference on Indoor Positioning and
Indoor Navigation (IPIN), pp. 1-8. https://doi.org/10.1109/IPIN. 2016.7743677

16. Meyer Zu Borgsen S, Schöpfer M, Ziegler L, Wachsmuth S (2014) Automated Door Detection with a 3D-Sensor, In: 2014 Canadian Conference on Computer and Robot Vision, pp. 276-282. https://doi.org/10.1109/CRV.2014.44

17. Quintana Galera B, Prieto S, Adan A, Bosché F (2018) Door detection in 3D coloured point clouds of indoor environments. Autom Constr 85:146-166. https://doi.org/10.1016/j.autcon.2017.10. 016

18. Arduengo $M$, Torras $C$, Sentis $L$ (2020) Robust and adaptive door operation with a mobile robot

19. Ramalingam B, Yin J, Rajesh Elara M, Tamilselvam YK, Mohan Rayguru M, Muthugala MAVJ, Félix Gómez B (2020) A human support robot for the cleaning and maintenance of door handles using a deep-learning framework. Sensors. https://doi.org/ $10.3390 / s 20123543$

20. (Willow Garage Star Detector). http://pr.willowgarage.com/wiki/ Star-Detector

21. Zhou QY, Park J, Koltun V (2018) Open3D: A Modern Library for 3D Data Processing. arXiv:1801.09847

22. Qi CR, Su H, Mo K, Guibas LJ (2016) PointNet: Deep Learning on Point Sets for 3D Classification and Segmentation. CoRR arXiv: abs/1612.00593

23. Wu H, Zhang J, Huang K, Liang K, Yizhou Y (2019) FastFCN: Rethinking Dilated Convolution in the Backbone for Semantic Segmentation, In arXiv preprint arXiv:1903.11816

24. Chao P, Kao CY, Ruan YS, Huang CH, Lin YL (2019) HarDNet: A low memory traffic network, arXiv:abs/1909.00948

25. Zhang H, Dana KJ, Shi J, Zhang Z, Wang X, Tyagi A, Agrawal A (2018) Context encoding for semantic segmentation, CoRR arXiv:abs/1803.08904

26. Sarathy S, Tao A, Barker J (2016) Detectnet: Deep neural network for object detection in digits. https://developer.nvidia.com/ blog/detectnet-deep-neural-network-object-detection-digits/

27. Badrinarayanan V, Kendall A, Cipolla R (2017) SegNet: a deep convolutional encoder-decoder architecture for image segmentation. IEEE Trans Pattern Anal Mach Intell 39:2481-2495

28. Yu C, Wang J, Peng C, Gao C, Yu G, Sang N (2018) BiSeNet: Bilateral segmentation network for real-time semantic segmentation. CoRR arXiv:abs/1808.00897

29. (Computer Vision Annotation Tool: A Universal Approach to Data Annotation). https://github.com/opencv/cvat

30. Crivellaro A, Rad M, Verdie Y, Moo Yi K, Fua P, Lepetit V (2015) A Novel Representation of Parts for Accurate 3D Object Detection and Tracking in Monocular Images, In Proceedings of the IEEE International Conference on Computer Vision (ICCV)

31. Lin T, Maire M, Belongie SJ, Bourdev LD, Girshick RB, Hays J, Perona P, Ramanan D, Dollár P, Zitnick CL (2014) Microsoft COCO: Common Objects in Context. CoRR arXiv:abs/1405.0312

32. Szegedy C, Vanhoucke V, loffe S, Shlens J, Wojna Z (2015) Rethinking the inception architecture for computer vision. CoRR abs/1512.00567. http://arxiv.org/abs/1512.00567

33. He K, Zhang X, Ren S, Sun J (2015) Deep residual learning for image recognition

Publisher's Note Springer Nature remains neutral with regard to jurisdictional claims in published maps and institutional affiliations. 